\title{
artigo
}

Sousa Silva, F.A.; Pereira, M.C.L.; Dias, M.V.S.; Cipriano, R.R.L.C.S.; Moura, S.G.; Rodrigues, A.C.E.;

Óbitos por cisticercose na região nordeste do Brasil em 2019

DOI: https://doi.org/10.36489/saudecoletiva.2021v11i65p6044-6053

\section{Óbitos por cisticercose na região nordeste do Brasil} em 2019

\author{
Cysticercosis deaths in the northeast region of Brazil in 2019
}

Muertes por cisticercosis em la región noreste de Brasil em 2019

\section{RESUMO}

O estudo teve como objetivo realizar um levantamento de dados epidemiológicos acerca da prevalência de infecções por cisticercose em seres humanos na região Nordeste do Brasil, no ano de 2019. 0 estudo foi feito com base nos dados disponibilizados pelo SINAN-DATASUS, sobre o tema. O maior número de óbitos foi encontrado no estado do Ceará. Os óbitos ocorreram em sua maioria em pacientes do sexo masculino, da faixa etária de 40 a 59 anos, com nenhum ano de escolaridade. Conclui-se que a persistência dos casos está ligada não apenas a penetrância e magnitude da doença, mas sim ao seu negligenciamento por parte das autoridades em saúde e a falta de políticas de saneamento básico para a população.

DESCRITORES: Cisticercose; Epidemiologia; Parasitologia; Morte.

\section{ABSTRACT}

The study aimed to carry out a survey of epidemiological data on the prevalence of infections by cysticercosis in human beings in the northeastern region of Brazil, in the year 2019. The study was made based on the data provided by SINAN-DATASUS, on the subject. The highest number of deaths was found in the state of Ceará. The deaths occurred mostly in male patients, aged 40 to 59 years, with no schooling years. It can be concluded that the persistence of the cases is linked not only to the penetration and magnitude of the disease, but to its neglect on the part of the health authorities and the lack of basic sanitation policies for the population.

DESCRIPTORS: Cysticercosis; Epidemiology; Parasitology; Death.

\section{RESUMEN}

El estudio tuvo como objetivo realizar una encuesta de datos epidemiológicos sobre la prevalencia de infecciones por cisticercosis en seres humanos en la región nororiental de Brasil, en el año 2019. El estudio se realizó con base en los datos proporcionados por SINAN-DATASUS, sobre el tema. El mayor número de muertes se encontró en el estado de Ceará. Las muertes ocurrieron principalmente en pacientes varones, de 40 a 59 años, sin años de escolaridad. Se puede concluir que la persistencia de los casos está ligada no solo a la penetración y magnitud de la enfermedad, sino a su descuido por parte de las autoridades de salud y la falta de políticas de saneamiento básico para la población.

DESCRIPTORES: Cisticercosis; Epidemiología; Parasitología; Muerte.

RECEBIDO EM: 30/01/2021 APROVADO EM: 16/02/2021

\section{Felipe André Sousa Silva}

Acadêmico de Medicina; Centro Universitário UNIFACID.

ORCID: 0000-0003-2317-6378

\section{Maria Clara Leal Pereira}

Acadêmico de Medicina; Centro Universitário UNIFACID.

ORCID: 0000-0002-2544-6733

\section{Maria Victoria Sousa Dias}

Acadêmico de Medicina; Centro Universitário UNIFACID.

ORCID: 0000-0002-2943-1934 
Ruth Ravena Luz Carvalho Sousa Cipriano

Acadêmico de Medicina; Centro Universitário UNIFACID.

ORCID: 0000-0002-1312-3048

\section{Sâmia Gonçalves de Moura}

Acadêmico de Medicina; Centro Universitário UNIFACID.

ORCID: 0000-0001-9640-5181

Augusto Cesar Evelin Rodrigues

Docente do Centro Universitário UNIFACID.

ORCID: 0000-0001-7469-981X

\section{INTRODUÇÃO}

A mplamente conhecido na literatura médica, o complexo teníase-cisticercose é considerado um grupo de zoonoses prevalente no Brasil, com altas taxas de infecções anualmente em nosso pais, especialmente, na região Nordeste, devido a precariedade das condições de vida desta população.

Essa patologia apresenta-se como um problema de saúde pública, uma vez que é capaz de atingir um grande número de pessoas vulneráveis a este tipo de infecção, seja pelo quadro clínico que pode desencadear, seja pelos óbitos que pode proporcionar. $^{1}$

O homem atua como o único hospedeiro definitivo, tanto para a Taenia solium como para a T. saginata e também como hospedeiro intermediário, juntamente com os suínos, para a T. solium, desenvolvendo a cisticercose em seus tecidos, especialmente a neurocisticercose, que é a forma mais grave e frequente desse complexo no ser humano ${ }^{2}$. Essa patologia apresenta pleomorfismo em sua clínica, pois o cisticerco tem a capacidade de se alojar em multiplos tecidos do hospedeiro, no entando apresenta predileção pelos tecidos muscular esqueléticos e nervoso, o que implica inúmeras repercussões clínicas ${ }^{2,3,14}$.

Nesse panorama, ao alojar-se na musculatura ou em tecido subcutâneo a larva pode apresentar poucos sintomas, uma vez que ocorre inflamação e formação de uma membrana adventícia fibrosa seguida da calcificação. Isso pode causar dor moderada, cãibras e fadiga. Quando se instala no miocárdio, pode levar a arritmias cardíacas. Junto a isso, há também a neurocis-
Amplamente

conhecido na

literatura médica, o

complexo teníase-

cisticercose é

considerado um

grupo de zoonoses

prevalente no

Brasil, com altas

taxas de infecções

anualmente em nosso

pais, especialmente,

na região

Nordeste, devido

a precariedade das

condições de vida

desta população. ticercose, que é apresentação clínica mais grave. $\mathrm{O}$ cisticerco se instala no parênquima nervoso, podendo levar a compressão nervosa, vasculopatias e morte neuronal. As manifestações clinicas mais comuns são: cefaleia, convulsões, distúrbios psíquicos e crises de ausência ${ }^{4,5}$.

Por estar relacionada a precárias condicções de vida a Organização Mundial de Saúde (OMS), entre 2008 e 2015, incluiu o complexo teníase/cisticercose no subgrupo de zoonoses negligenciadas, visando ao controle das chamadas doenças tropicais negligenciadas ${ }^{6,7}$. Nessa ótica, o estudo visou a realizar um levantamento epidemiológico dos óbitos causados por cisticercose humana na região Nordeste no ano de 2019. Tal busca se faz relevante devido ao caráter social que esta doença possui, uma vez que é categorizada como negligenciada, logo, buscar dados consolidados que evidenciem o real quadro dessa doença na região Nordeste irá expandir a literatura acerca do tema, bem como proporcionar visibilidade e maiores investimentos na prevenção, no controle e no tratamento deste agravo.

\section{MÉTODO}

Trata-se de um estudo descritivo, epidemiológico transversal, analítico, documental, realizado a partir dos dados obtidos pela consulta do SINAN-DATASUS, que é uma base de dados epidemiológicos, em meio eletrônico e as fontes consultadas para o embasamento teórico foram retiradas de bases de dados online, como PUBMED e SciELO. Os dados consolidados são de domínio público, o que permite que estes sejam consultados por qualquer pesquisador. 


\section{artigo}

Sousa Silva, F.A.; Pereira, M.C.L.; Dias, M.V.S.; Cipriano, R.R.L.C.S.; Moura, S.G.; Rodrigues, A.C.E.;

Óbitos por cisticercose na região nordeste do Brasil em 2019

Os resultados foram coletados no mês de dezembro de 2020. Os casos pesquisados foram dos nove estados que compõem a região nordeste do Brasil, relativos ao período temporal de um ano ( 2019 ), através das bases de dados PUBMED e SciELO, observando-se as seguintes variáveis: faixa-etária, gênero e anos de escolarização. Foram incluídos todos os óbitos ocorridos e registrados no SINAN-DATASUS, nos nove estados que compõem a região nordeste ( Maranhão, Piauí, Ceará, Rio Grande do Norte, Paraíba, Pernambuco, Sergipe, Alagoas e Bahia ). Foram excluídos do estudo, os casos registrados no SINAN-DATASUS, em que os pacientes eram de outras regióes do país. Após o levantamento dos dados, os mesmos foram analisados através de números absolutos e relativos $\mathrm{e}$ proporção na base de 100 e os resultados foram apresentados através de tabelas e gráficos para melhor compreensão.

Devido a isso, o estudo não necessitou da aprovação do Comitê de Ética e Pesquisa (CEP), contudo, os pesquisadores comprometeram-se a zelar pelos principios dipostos na Resolução No 510, de 7 de abril de 2016.

\section{RESULTADOS}

A Tabela.1 mostra os óbitos por cisticercose humana por Região/Unidade da Federação, segundo a faixa etária em 2019. A partir destes dados é possível inferir que as faixas etárias com maior acometimento foram as de 40 a 49 anos - 06 óbitos (27,27\%) e de 50 a 59 anos 06 óbitos (27,27\%).
Segundo a Tabela.2 é possível observar a relaçao entre o nível educacional e a quantidade de óbitos por cisticercose humana na região nordeste do Brasil em 2019. O maior número de obitos ocorreu entre os que possuíam menor tempo de escolaridade, com um total de seis óbitos $(27,27 \%)$. No entanto, há um distribuição regular de 4 óbitos $(18,12 \%)$ para as demais faixas de anos de escolaridade.

Conforme a Tabela.3, a distruibuição dos óbitos por Região/Unidade da Federação segundo residência e sexo, concentrou-se na população masculina com um total de 15 óbitos $(68,18 \%)$, o que é mais da metade se comparados ao número absoluto de óbitos femininos, que foram de 7 (31,81\%). Do total de óbitos, 7 (31,81\%) eram residentes no estado do Ceará, cujo

\section{TABELA 1. Óbitos por cisticercose humana, segundo faixa etária e Região/Unidade da Federação. Brasil. 2019.}

\section{REGIÃO UNIDADE DA FEDERAÇÃO}

NORDESTE

MA

PI

CE

RN

PB

PE

BA

\section{FAIXA ETÁRIA}

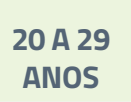

30 A 39
ANOS

40 A 49
ANOS
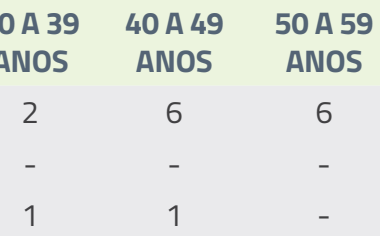

2

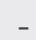

1
6
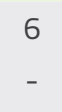

$$
1
$$

1

1

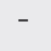

1

2

1

3
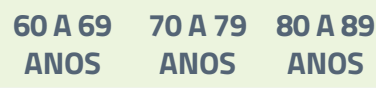

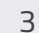

2

TOTAL

1

3

$-$

22

$-$

-

1

2

1

1

1

9

1

-

$-$

$-$

- 2

TABELA 2. Óbitos por cisticercose humana, segundo nível de escolaridade e

Região/Unidade da Federação. Brasil. 2019.

\section{REGIÃO/UNIDADE DA} FEDERAÇÃO

Região Nordeste
Maranhão
Piauí
Ceará

Rio Grande do Norte Paraíba

Pernambuco Bahia

\section{BRANCA} PRETA

PARDA

IGNORADO

TOTAL

-

$\begin{array}{ll}2 & 1 \\ - & - \\ - & - \\ 1 & -\end{array}$

19

1

1

8

2

1

1

5
22

1

1

9

2

1

1

7

Fonte: MS/SVS/CGIAE - Sistema de Informações sobre Mortalidade - SIM.

número de óbitos é quase o dobro do estado com o segundo maior número de óbitos, a Bahia, com 4 óbitos (18,18\%).

A Tabela.4 mostra os óbitos por Região/ Unidade da Federação, segundo a cor/raça, onde o maior número de óbitos por cisticercose humana concentrou-se na população auto-declarada parda, 19 óbitos, que representa cerca de $86,36 \%$ em números relativos. Tais dados estão concordantes com os estudos epidemiológicos que evedenciam que as taxas de infecção e óbitos são as maiores dentre o maior contingente populacional, que corresponde a pardos maoioria étinca brasileira - e aos dados pro- 
TABELA 3. Óbitos por residência por sexo segundo Região/Unidade da Federação. Brasil. 2019.

\section{REGIÃO UNIDADE DA FEDERAÇÃO}

NORDESTE

MA

$\mathrm{PI}$

CE

RN

PB

PE

BA

\section{NENHUMA}

6

$-$

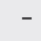

2

1

1

- 1

2

1

1

1 A 3
ANOS

$\begin{array}{ll}4 \text { A } 7 & 8 \text { A } 11 \\ \text { ANOS } & \text { ANOS }\end{array}$

4

4

IGNORADO TOTAL

$-$

$-$

1

4

- 1

2

1

4

4

22

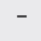

1

2

$-$

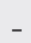

$-$

1

-

$-$

1

1

9

2

1

1

7
Fonte: MS/SVS/CGIAE - Sistema de Informações sobre Mortalidade - SIM

\section{TABELA 4. Óbitos por residência por cor/raça segundo Região/Unidade da} Federação. Brasil. 2019

$\begin{array}{cccc}\text { REGIÃO/ UNIDADE DA FEDERAÇÃO } & \text { MASCULINO } & \text { FEMININO } & \text { TOTAL } \\ \text { Região Nordeste } & 15 & 7 & 22 \\ \text { Maranhão } & - & 1 & 1 \\ \text { Piauí } & - & 1 & 1 \\ \text { Ceará } & 7 & 2 & 9 \\ \text { Rio Grande do Norte } & 2 & - & 2 \\ \text { Paraíba } & 1 & - & 1 \\ \text { Pernambuco } & 1 & - & 1 \\ \text { Bahia } & 4 & 3 & 7\end{array}$

venientes dos recenciamentos nacionais, em que estes afirmam que a maior parte da população brasileira é composta de indivíduos não-brancos (pardos, pretos) $)^{12,14 .}$

Dos óbitos ocorridos no Ceará (09 obitos), apenas 01 óbito ocorreu em brancos $(11,11 \%)$ e $08(88,88 \%)$ em pardos e dos 07 óbitos ocorridos na Bahia, 01 (14,66 $\%)$ em brancos, 01 (14,66 \%) em pretos e 05 (70,78\%) em pardos. No Maranhão, Piauí, Paraíba e Pernambuco, todos os casos ocorreram em pardos.

\section{DISCUSSÃO}

É possível observar na região, que o estado que possuiu o maior número de óbitos foi o Ceará com 09 óbitos (40,0\%). Este estado também foi o único que apresentou morte na faixa etária de 20 a 29 anos: 02 óbitos (9,09\%). Isto pode estar associado a um maior estado de vulnerabildiade de sua população, justificando-se, assim, a quantidade de desfechos fatais da doença.

Estudos evidenciaram que a cisticercose é mais comum em pacientes com mais de 40 a 60 anos de idade, o que é corroborado pelos dados obtidos a partir do SINAN-DATASUS. Isto ocorre devido ao tempo de incubação e manifestação dos sintomas ser longo, haja vista que a infecção pela ingestão de ovos de Taena solium geralmente se dar nas faixas etárias menores (infância e adolescência), e o diagnóstico e aparecimento dos sintomas e os óbitos vão se dar nas faixas etárias maiores (acima dos 40 anos) $)^{9}$. Quanto à maioria dos casos terem ocorridos no estado do Ceará, pode-se associar a uma pior condição de saúde dessa população ou mesmo a uma melhoria do sistema de saúde, no sentido de diagnósticar essa patologia em relação aos demais estados da região ${ }^{1}$.

Como a maior parte dos óbitos ocorreu em indivíduos em idade laboral, isso representa um grande prejuízo ao cenário de contribuição e renda, pois são indivíduos economicamente ativos.

Quando associamos os óbitos com a escolaridade, percebemos que os mesmos refletem a vulnerabilidade das pessoas de menor escolaridade, uma vez que indivíduos com menor escolaridade buscam menos os serviços de saúde. ${ }^{14}$. Quanto aos casos na parcela da população de maior escolaridade, pode-se justificar, apesar da doença ser transmitida através da ingestão de ovos viáveis de T. solium, pelas diversidades de formas de contágio, como pela água, alimentos, mãos sujas ou retroperistaltismo ${ }^{9,12,14}$. O estado do Ceará apresentou óbitos entre todas as faixas de escolaridade, 2 óbitos (9,09\%) em pessoas sem nenhuma escolaridade, 1 óbito $(4,5 \%)$ na faixa de 1 a 3 anos de escolaridade e nos demais níveis de escolaridade, apresentou 2 óbitos (9, 09\%) em cada uma, respectivamente. Tais dados podem sugerir precários indicadores de saúde no estado, bem como difícil acesso aos serviços de saúde ${ }^{1}$.

O complexo teníase-cisticercose possui sua distribuição de caráter cosmopolita e isso contribui para que este se torne um problema de saúde pública em localidades que possuem precárias condições sanitárias e socioeconômica, que ocasionam sua perpetuação. Isso é observado quanto ao maior número de óbitos por cisticercose em indivíduos com menor grau de escolaridade $^{1,8}$, além de limitar a possibilidade de exportação de carnes ${ }^{8,12}$.

Os resultados discordam de outras pesquisas em que a maior parte dos individuos acometios pela doença eram do sexo feminino. Contudo, devido aos estigmas sociais apresentados pelos homens em buscarem serviços médicos, é possível inferir que os desfechos fatais estejam ligados a não busca de serviços de saúde em tempo hábil, ou ao total desconhecimento dos sinais e sintomas da doença, ${ }^{9,13,14}$.

Existe uma disparidade muito grande 
entre o número de casos e as etnias, revelando uma predominância muito grande de óbitos entre as pessoas de cor/raça parda. Historicamente no Brasil, indivíduos brancos apresentam melhores níveis de vida e acesso aos serviços de saúde e saneamento básico, diferente de indivíduos não-brancos que não possuem acesso aos serviços de saúde e saneamento básico, nem mesmo instrução, quando comparados aos indivíduos brancos ${ }^{9,10,14}$. Associado a isso, essa parcela da população é numericamente superior às outras no Brasil, segundo dados do IBGE, o que poderia justificar esses achados.

\section{CONCLUSÃO}

O complexo teníase-cisticercose possui caráter cosmopolita, ou seja, está ligado às atividades humanas, visto que o ser humano é o hospedeiro definitivo da tênia, em sua forma adulta. Nesse panorama, o complexo teníase-cisticercose permanece com elevados índices no Nordeste, com

\section{O complexo teníase-}

cisticercose possui

caráter cosmopolita,

ou seja, está ligado

às atividades

humanas, visto

que o ser humano

é o hospedeiro

\section{definitivo da tênia,} em sua forma adulta.

destaque para o estado do Ceará, visto que este estado apresentou nove óbitos por cisticercose, o que representa maior grau de vulnerabilidade de sua população. Nessa ótica, o complexo teníase-cisticercose segue como uma doença tropical negligenciada, infecta pessoas pobres, de baixa escolaridade, que não tem acesso aos serviços de saúde primários e não possuem saneamento básico. Isto é potencializado pela falta de água, o que força esses indivíduos a consumirem água de fontes não tratadas, o que fomenta o ciclo da infecção.

Além disso, regiões interioranas possuem criação de animais e consumo de alimentos não vistoriados, fato que contribui para a perpetuação dos ciclos infecciosos. No entanto, a gênese da ciclicidade infecciosa do complexo teníase-cisticercose é de natureza social e, portanto, para evitar sua propagação, é necessária uma maior atenção das autoridades, campanhas de proteção a saúde para instruir a população acerca deste complexo, bem como obras governamentais que ofertem serviços de saneamento básico e condições de moradia humanamente dignas à população.

\section{REFERÊNCIAS}

1. Bomtempo, Priscila Taciani, et al. "Impacto da cisticercose na produção de carnes bovina e suína." Pubvet 12 (2018): 136.

2. Brito, Katiane Rodrigues de. "Teníase e cisticercose: aspectos clínicos e epidemiológicos." (2018).

3. Brasil, Ministério da Saúde. Banco de dados do Sistema Único de Saúde-DATASUS. Disponível em http://tabnet.datasus.gov.br/ cgi/deftohtm.exe?sim/cnv/obt10uf.def [acessado em 25 de janeiro de 2021].

4. Chieffi, Pedro Paulo, and Sergio Vieira dos Santos. "Teníasecisticercose: uma zoonose negligenciada/Taeniasis-cysticercosis: a neglected zoonosis." Arquivos Médicos dos Hospitais e da Faculdade de Ciências Médicas da Santa Casa de São Paulo 65.1 (2020): 1-8.

5. Da Silva Targino, Gizia et al. ASPECTOS CLÍNICOS E EPIDEMIOLÓGICOS DA TENÍASE. Mostra Interdisciplinar do curso de Enfermagem, v. 2, n. 2, 2017.

6. Da Silva, Priscilla Elias Ferreira, Fernando Lourenço Pereira, and Catarina Teixeira. "O complexo teníase/cisticercose abordado em um jogo didático para o ensino de ciências." (2016).

7. Garro, F. L. et al. Diagnóstico do complexo teníase-cisticercose bovina em São João Evangelista, Minas Gerais, Brasil. Arquivo Brasileiro de Medicina Veterinária e Zootecnia, v. 67, n. 4, p. 10631069, 2015.
8. Magalhães, Fernanda C., et al. "Diagnóstico e fatores de risco do complexo teníase-cisticercose bovina no município de Salinas, Minas Gerais." Pesquisa Veterinária Brasileira 37.3 (2017): 205-209.

9. Muniz, Ana Paula Mendes et al. Análise retrospectiva dos casos de cisticercose humana do Hospital de Clínicas da Universidade Federal de Uberlândia-MG, no período de 2000-2017. 2018.

10. Rosa, Ruy Roberto Porto Ascenso, et al. "PERFIL EPIDEMIOLÓGICO DA CISTICERCOSE NO MUNICÍPIO DE CAJUEIRO DA PRAIA, PIAUÍ-BRASIL/EPIDEMIOLOGY OF CYSTICERCOSIS IN CAJUEIRO DA PRAIA, PIAUÍ BRAZIL." Saúde em Foco 3.1 (2016): 146-159.

11. Santos, Karina Rodrigues, et al. "Conhecimento de feirantes de mercados municipais da cidade de Parnaíba-PI, sobre teníase-cisticercose." Revista Brasileira de Educação e Saúde 10.3 (2020): 79-84.

12. Soares, Thaigra. "CISTICERCOSE, UMA DOENÇA NEGLIGENCIADA, MAS NÃO ESQUECIDA: uma revisão." Revista Panorâmica online 19 (2017).

13. Targino, Gizia da Silva, et al. "Aspectos clínicos e epidemiológicos da teníase." (2016).

14. Toledo, Rômulo César Clemente et al. complexo teníase/cisticercose: uma revisão. Higiene Alimentar, v. 32, n. 282/283, p. 31-34, 2018. 